\title{
Calculation of Solid Solubility of Complex Molecules in Supercritical Carbon Dioxide using a Solution Model Approach
}

\author{
JAW-SHIN CHENG, MUOI TANG* and YAN-PING CHEN ${ }^{\dagger}$ \\ Department of Chemical Engineering, National Taiwan University, Taipei, Taiwan
}

(Received October 2002; In final form November 2002)

Solid solubility of complex molecules in supercritical carbon dioxide was calculated using a solution model approach. These solutes include the biological compounds of antioxidants, steroids, vitamins, and heavy aromatics. The modified AD (m-AD) activity coefficient model proposed in our previous study [Cheng, J.S., Tang, M. and Chen, Y.P. "Correlation and comparison of the infinite dilution activity coefficient on aqueous mixtures from a modified excess free energy model" Fluid Phase Equilib. (2002) Submitted for publication] coupled with the Flory-Huggins equation was employed. The molar volume of the solutes in the supercritical phase and the solid-fluid interaction parameter were optimally fitted for each solid component. Satisfactory results were obtained from the m-AD model with three or four parameters. The overall accuracy in this study is comparably good to that from the conventional equation of state method or the semi-empirical correlation equation. This result suggests a feasible high-pressure solid solubility calculation with the application of a theoretically based solution model.

Keywords: Method of calculation; Solid-fluid equilibria; Biological compounds; Supercritical carbon dioxide

\section{INTRODUCTION}

The application of supercritical technology has received much attention in the past two decades, especially in the field of the extraction and purification of pharmaceuticals, food and natural products. Experimental solubility data as well as the corresponding thermodynamic correlations are therefore essential to the proper chemical process design. Conventionally, the equation of state (EOS) method is applied to calculate the solid solubility in supercritical fluids. Recently, Ashour et al. [2] have presented the comparison of several EOS and mixing rules on the solid solubility calculation in supercritical phase. In the EOS approach, the sublimation pressure and the critical properties of the solute are required. For complex solids, the EOS method is usually limited due to these uncertain data. An alternative is the application of the solution model. Iwai et al. [3] have correlated the solubility of aromatic, and heavy alcohol and acid compounds in supercritical $\mathrm{CO}_{2}$ using a modified regular solution model. An extension was also presented in our previous study [4] on evaluating the solubility of biological compounds with acceptable accuracy. Other activity coefficient models are demonstrated in literature. For example, Bush and Eckert [5] have proposed a predictive linear solvation energy relationship (LSER) on solid solubility calculation. Relatively large errors are shown using the predictive method. For engineering purpose, a satisfactory correlation from a theoretically based model is desired in process design and calculation.

Recently, Aranovich and Donohue (AD) [6] developed a lattice-based internal energy model, which took the quasi-chemical theory and the local composition effect into account. It was demonstrated

*Present Address: Department of Chemical Engineering, Chinese Culture University, Taipei, Taiwan.

${ }^{\mathrm{t}} \mathrm{z}$ Corresponding author. Fax: +886-2-2362-3040. E-mail: ypchen@ccms.ntu.edu.tw 
that the AD model yielded good agreement with the molecular simulation data, especially at infinite dilution or high-density condition. With an improved expression for the local composition, a modified excess Gibbs free energy model (m-AD model) was derived in our previous study [1]. Satisfactory results of the infinite dilution activity coefficients on aqueous and organic mixtures were reported using the m-AD model. In this study, the m-AD model coupled with the Flory-Huggins equation was employed to evaluate the solid solubility of the biological and heavy aromatic compounds in supercritical $\mathrm{CO}_{2}$. Comparison of the calculated solid solubility with those from the EOS approach and several semi-empirical correlation equations are also presented.

\section{METHOD OF CALCULATIONS}

Applying the solution model, the supercritical solvent is treated as an expanded liquid. The equilibrium solubility of a solid solute (component 2) in a supercritical fluid (component 1 ) is expressed as:

$$
y_{2}=f_{2}^{s} / \gamma_{2} f_{2}^{l}
$$

The activity coefficient is represented by its limiting value at infinite dilution for the low solubility of the complex molecules. In this study, the m-AD model is employed to express the residual part of infinite dilution activity coefficient while the Flory-Huggins equation is used for the combinatorial contribution. The derivation of the m-AD model was presented in our previous work [1]. Basically, this model discussed the distribution probability of molecules in lattice sites. Local composition expressions were then formulated and thermodynamic properties were evaluated for a fixed coordination number of lattice fluid. The m-AD model was developed based on a theoretically consistent approach with respect to the molecular simulation results of both monomer and polymer systems. Application of this model to solid solubility calculations in supercritical fluid is also expected to yield satisfactory accuracy. The complete limiting activity coefficient from the residual part of the m-AD model coupled with the Flory-Huggins combinatorial term is:

$$
\begin{aligned}
\ln \gamma_{2}^{\infty}= & \ln \gamma_{2}^{\infty, C}+\ln \gamma_{2}^{\infty, R} \\
= & {\left[\ln \left(\frac{v_{2}}{v_{1}}\right)+1-\frac{v_{2}}{v_{1}}\right]-\frac{z}{4} q_{2} } \\
& \times\left\{\frac{1}{c-5}\{\exp [-(c-5) \Delta / R T]-1\}-\frac{\Delta}{R T}\right\}
\end{aligned}
$$

where $v$ is the molar volume, $\Delta$ is the solid-fluid interaction energy parameter in the m-AD model, and the coefficient $c$ equals to $2^{-6}+4(\sqrt{2})^{-6}$. The coordination number $z$ is six since a simple cubic lattice is approximated in the m-AD model. Incorporating the infinite dilution activity coefficient and neglecting the difference in heat capacities of solute in the solid and the supercritical phases, the solubility of a solid in the supercritical fluid is:

$$
\begin{aligned}
\ln y_{2}= & \left(\Delta H_{2}^{f} / R\right)\left(1 / T_{2, m}-1 / T\right)-1+\left(v_{2} / v_{1}\right)-\ln \left(v_{2} / v_{1}\right) \\
& +\frac{z}{4} q_{2}\left\{\frac{1}{c-5}\{\exp [-(c-5) \Delta / R T]-1\}-\frac{\Delta}{R T}\right\}
\end{aligned}
$$

where $\Delta H_{2}^{\mathrm{f}}$ and $T_{2, \mathrm{~m}}$ are the heat of fusion and the melting temperature of the solid solute, respectively. The fusion heat was either taken from the literature or estimated by the method of Yalkowsky [7]. With the application of the Jacobsen and Stewart EOS with 32 constants regressed by Ely et al. [8], the molar volume $v_{1}$ of supercritical $\mathrm{CO}_{2}$ was determined. In this study, the molar volume $v_{2}$ of the solute in supercritical phase, and the interaction energy parameter $\Delta$ were taken as adjustable parameters. They were regressed for each solid by minimizing the following objective function:

$$
\mathrm{obj}=\sum\left|y_{2 i}^{\mathrm{cal}}-y_{2 i}^{\exp }\right| / y_{2 i}^{\exp }
$$

where $y_{2}^{\text {cal }}$ and $y_{2}^{\text {exp }}$ are the calculated and experimental solid solubility in supercritical $\mathrm{CO}_{2}$. The summation is running over all experimental data points $i$.

To compare the solid solubility calculations in supercritical fluid, EOS method and semi-empirical correlation equation are also presented in this study. In the conventional EOS approach, the PengRobinson (PR) EOS with the van der Waals 2 (vdW2) mixing rules and two binary interaction parameters were employed. The sublimation pressures of the complex molecules are usually unavailable, hence they are also considered as adjustable parameters. In the semi-empirical correlation method, equations based on an association law [9], the theory of dilute solutions [10], and the equation derived from the formation and reaction equilibrium of the solvent-solute clusters [11] were all employed for the comparative study of the solid solubility calculations.

\section{RESULTS AND DISCUSSION}

In this study, the m-AD model together with the Flory-Huggins equation was employed to evaluate 
TABLE I Optimally fitted parameters for the calculation of the solid solubility in supercritical $\mathrm{CO}_{2}$ using the m-AD model

\begin{tabular}{|c|c|c|c|c|c|c|c|}
\hline \multirow[b]{3}{*}{ Solid solute } & \multicolumn{7}{|c|}{ Parameters of the m-AD model } \\
\hline & \multicolumn{3}{|c|}{ Three-parameter method } & \multicolumn{4}{|c|}{ Four-parameter method } \\
\hline & $\Delta$ & $\alpha$ & $\beta$ & $\Delta_{0}$ & $\Delta_{1}$ & $\alpha$ & $\beta$ \\
\hline 9,10-Anthraquinone & 788.87 & -8.711 & -50.37 & 769.88 & 0.880 & -8.573 & -49.71 \\
\hline Ascorbic acid & 980.85 & -6.026 & -39.52 & 1443.4 & -29.92 & -6.019 & -39.83 \\
\hline Ascorbyl palmitate & 365.82 & -13.55 & -66.90 & 1064.5 & 3.795 & -10.59 & -90.12 \\
\hline 1,4-Benzoquinone & 912.61 & -0.345 & -9.128 & 780.73 & -0.361 & -5.367 & -35.44 \\
\hline Cholesterol & 437.89 & -7.739 & -45.54 & 423.76 & 0.632 & -6.993 & -42.61 \\
\hline DDT & 857.03 & -7.192 & -44.73 & 890.90 & 1.757 & -6.217 & -42.42 \\
\hline 2,4-Dichlorophenoxy-acetic acid & 1076.7 & -7.134 & -41.86 & 1076.0 & 1.474 & -5.633 & -36.30 \\
\hline Dodecyl gallate & 553.68 & -8.169 & -45.77 & 701.69 & 1.128 & -6.836 & -45.75 \\
\hline Flavone & 783.42 & -4.853 & -33.59 & 849.24 & 1.984 & -4.277 & -33.24 \\
\hline Hexachlorobenzene & 1273.7 & -9.340 & -49.49 & 1281.4 & -0.393 & -9.742 & -51.20 \\
\hline 3-Hydroxy flavone & 733.90 & -3.630 & -27.86 & 821.85 & 1.547 & -3.229 & -28.72 \\
\hline Medroxyprogesterone acetate & 390.49 & -11.08 & -57.33 & 382.19 & 0.661 & -9.144 & -49.73 \\
\hline 1,4-Naphthoquinone & 889.45 & -5.204 & -35.92 & 1203.9 & 2.934 & -0.727 & -9.925 \\
\hline Naproxen & 851.82 & 0.815 & -4.818 & 861.26 & 1.321 & 0.092 & -7.562 \\
\hline Progesterone & 559.28 & -10.61 & -58.47 & 537.28 & 0.603 & -9.958 & -55.54 \\
\hline Propyl gallate & 818.76 & -7.940 & -43.90 & 798.71 & 0.620 & -6.915 & -39.60 \\
\hline Stigmasterol & 408.15 & -7.655 & -43.83 & 409.19 & 0.048 & -7.910 & -44.90 \\
\hline Testosterone & 663.11 & 0.506 & -5.664 & 662.93 & 0.369 & 0.381 & -6.139 \\
\hline Vitamin A & 425.32 & -14.24 & -70.11 & 484.89 & 1.980 & -7.199 & -45.13 \\
\hline Vitamin D2 & 381.57 & 0.465 & -6.157 & 382.12 & -0.098 & 0.582 & -5.707 \\
\hline Vitamin D3 & 350.88 & -10.03 & -52.57 & 747.27 & 2.278 & -6.351 & -55.03 \\
\hline
\end{tabular}

the solubility of the complex bio-molecules and heavy aromatics in supercritical $\mathrm{CO}_{2}$. Iwai et al. [3] and our previous study [4] have shown that the molar volumes of the solute components in the supercritical phase $v_{2}$ were adjustable parameters in the calculation. On applying the m-AD model to solid solubility calculation in supercritical $\mathrm{CO}_{2}$, both $v_{2}$ and $\Delta$ are evaluated through data regression. Similar to our previous research [4], it was again observed that the optimally fitted logarithm values of $v_{2}$ vary linearly with the logarithm of solvent density $\rho_{1}$. A simple correlation is then proposed:

$$
\ln v_{2}=\alpha \ln \rho_{1}+\beta
$$

where $\alpha$ and $\beta$ are two temperature independent constants for each solid solute and are obtained by regressing the experimental solubility data. In the m-AD model, the interaction energy parameter $\Delta$ between each solid solute and supercritical $\mathrm{CO}_{2}$ pair is taken as the third adjustable parameter. If the energy parameters are treated as temperatureindependent, the best-fitted values of these three parameters are listed in Table I for all binary systems investigated. Since the solid solubility data were correlated at various isotherms, an alternative way is to consider a temperature dependent form of the energy parameter:

$$
\Delta=\Delta_{0}+\Delta_{1} \times\left(T-T_{\text {ref }}\right)
$$

where $T_{\text {ref }}$ is the reference temperature at $298.15 \mathrm{~K}$, and $\Delta_{0}$ and $\Delta_{1}$ are two temperature independent constants. In this case, there are four parameters in the m-AD model, and their optimally fitted values are also presented in Table I.

The correlation results of the solid solubility for biological and heavy aromatic compounds using the m-AD model with three or four parameters are shown in Table II. It is indicated that with three parameters in the solution model approach, the overall absolute average deviation (AAD) on solid solubility is $13.79 \%$. This is slightly better than that reported in our previous study using two parameters in the modified regular solution model [4]. Upon taking the temperature dependent energy interaction parameters, the four-parameter m-AD model yields an improved AAD of $9.05 \%$ as shown also in Table II.

Figure 1 illustrated the calculated solubility of a heavy nonpolar aromatic compound 9,10-anthraquinone at 308.15 and $318.15 \mathrm{~K}$ in supercritical $\mathrm{CO}_{2}$ using either the three- or four-parameter m-AD model. Comparably good calculated AAD are obtained as 8.84 and $7.07 \%$, respectively. Another example is shown in Fig. 2 for a polar biological molecule stigmasterol at 308.15 and $333.15 \mathrm{~K}$. The m-AD model again yields similar results using either three or four parameters. For the biological compound cholesterol, the correlated results using the m-AD model are demonstrated in Fig. 3. The calculated AAD from the three- and four-parameter m-AD model are 10.25 and $2.25 \%$, respectively. Although the four-parameter method gives a very low calculated error, the three-parameter model is still within the experimental accuracy of the measured data. It is observed from Table II that the m-AD model shows the peak error around 20\%. This is satisfactory with respect to the accuracy of 
TABLE II Correlation results of the solid solubility for biological and heavy aromatic compounds using the m-AD model

\begin{tabular}{|c|c|c|c|c|c|c|c|}
\hline & \multirow[b]{2}{*}{ Solid solute } & \multirow[b]{2}{*}{ Temp. range $(K)$} & \multirow[b]{2}{*}{ Pressure range ( $\mathrm{MPa})$} & \multirow[b]{2}{*}{ Data points (NP) } & \multicolumn{2}{|c|}{$\operatorname{AAD}(\%)$} & \multirow[b]{2}{*}{ Data Ref. } \\
\hline & & & & & 3-Parameter method & 4-Parameter method & \\
\hline \multirow{4}{*}{ Antioxidants } & Ascorbic acid & 313.10 & $13.0-20.0$ & 4 & 1.99 & 1.99 & [12] \\
\hline & Ascorbyl palmitate & $308.10-313.10$ & $13.0-20.0$ & 8 & 9.43 & 3.64 & [12] \\
\hline & Dodecyl gallate & $313.10-333.10$ & $15.0-25.0$ & 8 & 7.27 & 4.24 & [12] \\
\hline & Propyl gallate & $313.10-333.10$ & $15.0-25.0$ & 8 & 6.77 & 1.18 & [12] \\
\hline \multirow[t]{3}{*}{ Pollutants } & DDT & $313.10-333.10$ & $10.4-20.8$ & 18 & 16.95 & 2.78 & [13] \\
\hline & 2,4-Dichlorophenoxy acetic acid & $313.10-333.10$ & $10.4-20.8$ & 16 & 11.89 & 2.35 & [13] \\
\hline & Hexachlorobenzene & $298.0-318.0$ & 11.2 & 4 & 0.20 & 0.00 & [14] \\
\hline \multirow[t]{5}{*}{ Steroids } & Cholesterol & $313.15-333.15$ & $10.0-25.0$ & 17 & 10.25 & 2.25 & [15] \\
\hline & Medroxyprogesterone acetate & $313.0-333.0$ & $11.0-22.0$ & 8 & 8.54 & 2.97 & [16] \\
\hline & Progesterone & $308.15-333.0$ & $9.0-24.4$ & 47 & 15.97 & 13.35 & {$[16,17]$} \\
\hline & Stigmasterol & $308.15-333.15$ & $9.1-30.4$ & 19 & 9.98 & 9.87 & {$[18]$} \\
\hline & Testosterone & $308.15-328.15$ & $8.7-24.3$ & 39 & 18.42 & 16.54 & {$[17]$} \\
\hline \multirow[t]{3}{*}{ Vitamins } & Vitamin A & $313.15-353.15$ & $20.0-35.0$ & 20 & 22.64 & 7.87 & [19] \\
\hline & Vitamin D2 & $313.15-353.15$ & $20.0-32.0$ & 17 & 9.91 & 9.84 & [19] \\
\hline & Vitamin D3 & $313.15-353.15$ & $20.0-35.0$ & 22 & 18.33 & 16.29 & [19] \\
\hline \multirow[t]{6}{*}{ Others } & 9,10-Anthraquinone & $308.15-318.15$ & $8.4-30.6$ & 17 & 8.84 & 7.07 & [20] \\
\hline & 1,4-Benzoquinone & $308.15-318.15$ & $8.6-29.2$ & 18 & 13.73 & 13.60 & [20] \\
\hline & Flavone & $308.20-318.20$ & $9.1-25.3$ & 10 & 17.04 & 13.62 & [21] \\
\hline & 3-Hydroxyflavone & $308.20-318.20$ & $9.1-25.3$ & 10 & 10.99 & 8.82 & [21] \\
\hline & 1,4-Naphthoquinone & $318.15-343.15$ & $10.1-36.4$ & 18 & 13.43 & 5.31 & [22] \\
\hline & Naproxen & $313.10-333.10$ & $9.0-19.3$ & 18 & 13.11 & 4.05 & [23] \\
\hline Grand & & & & 346 & 13.79 & 9.05 & \\
\hline
\end{tabular}

$\operatorname{AAD}(\%)=\frac{1}{N P} \sum_{i}\left|y_{2 i}^{\text {cal }}-y_{2 i}^{\exp }\right| / y_{2 i}^{\text {exp }}$. 


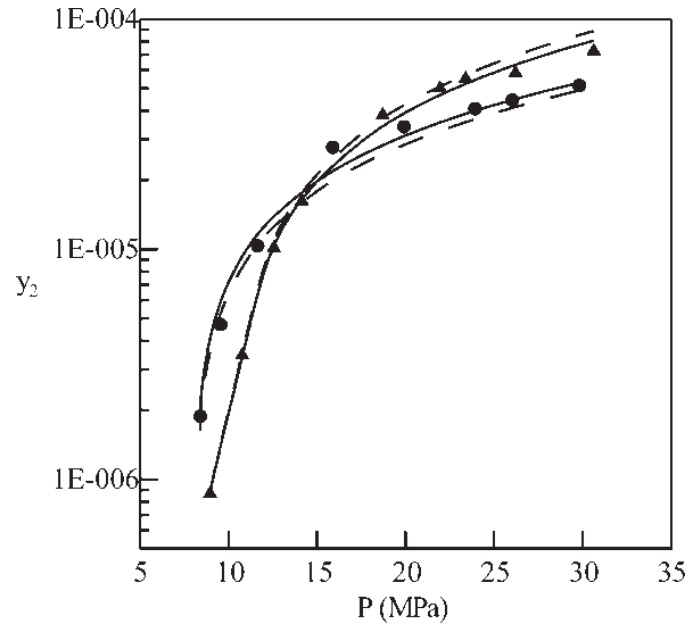

FIGURE 1 Comparison of the experimental and calculated solid solubility of 9,10-anthraquinone from the m-AD model. Experimental data: $(\bullet) 308.15 \mathrm{~K}$; (ム) $318.15 \mathrm{~K}$. Correlation results: (一) four-parameter method; (- - ) three-parameter method.

low solid solubility. For almost half of the systems listed in Table II, either the three- or four-parameter m-AD model yields comparable results.

For comparative purpose, the conventional EOS approach and the semi-empirical correlation equation were also employed in this study. The comparison of the overall deviations from various methods is demonstrated in Table III. Using the modified regular solution model in our previous study with two parameters [4], an AAD of $14.70 \%$ is obtained. For the $\mathrm{m}$-AD model with the Flory-Huggins equation using three and four parameters, the overall AAD are 13.79 and $9.05 \%$, respectively. Concerning the semi-empirical correlation equations, three to four parameters are usually required. A three-parameter equation proposed by Chrastil [9] was employed where an AAD of

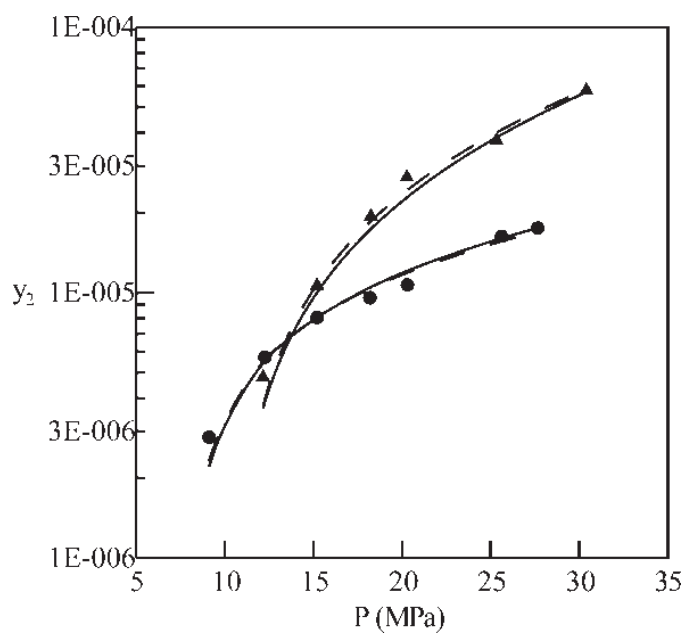

FIGURE 2 Comparison of the experimental and calculated solid solubility of stigmasterol from the m-AD model. Experimental data: $(\bullet)$ 308.15K; (ム) $333.15 \mathrm{~K}$. Correlation results: (一) fourparameter method; $(---)$ three-parameter method.

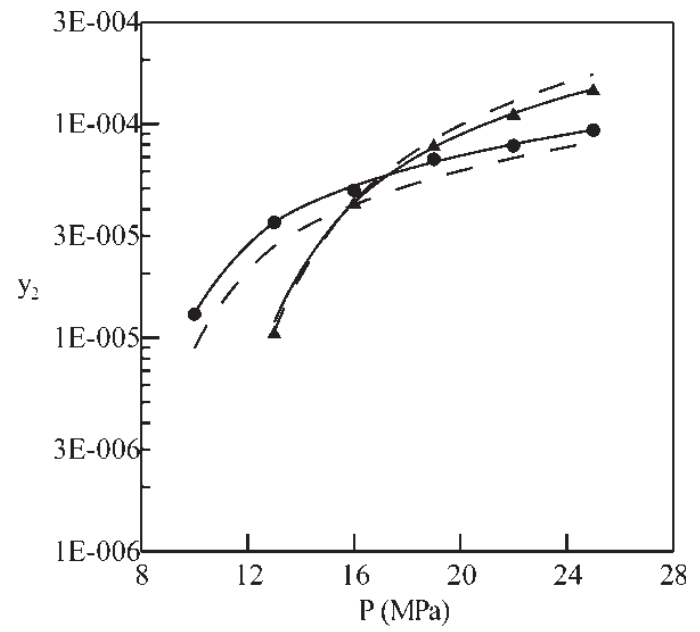

FIGURE 3 Comparison of the experimental and calculated solid solubility of cholesterol from the m-AD model. Experimental data: $(\bullet) 313.15 \mathrm{~K}$; ( $\mathbf{\Lambda}) 333.15 \mathrm{~K}$. Correlation results: (-) four-parameter method; (- - ) three-parameter method.

$10.93 \%$ was obtained. A similar result with an AAD of $11.01 \%$ was presented using another three-parameter equation derived by Méndez-Santiago and Teja [10]. With four parameters, the semi-empirical correlation equation developed by Jiang et al. [11] yields an AAD of $9.95 \%$. In the EOS approach, the PR EOS and vdW2 mixing rules were applied. The uncertain sublimation pressures of complex solids expressed in an Antoine type equation were also taken as adjustable parameters. Therefore, the EOS has four parameters that are evaluated by data regression. The grand deviation from the EOS method has an AAD of $10.16 \%$, as depicted in Table III. Generally, the m-AD model presents comparably good accuracy to that from the empirical correlation method with equal number of parameters. The m-AD model preserves reasonable theoretical basis, and presents not only satisfactory solid solubility but also other phase equilibrium calculation results. The m-AD model is recommended as a consistent and useful engineering approach for designing the separation and purification processes.

\section{CONCLUSION}

Solid solubility of complex biological and heavy aromatic compounds in supercritical $\mathrm{CO}_{2}$ was correlated in this study using the m-AD model. Comparison of the calculated results is also presented using the EOS approach and the semiempirical correlation equation. The m-AD model coupled with the Flory-Huggins equation with three or four parameters yields comparably satisfactory accuracy to those from the other approaches. The m-AD model, however, has a superior theoretical basis and provides a useful engineering 
TABLE III Comparison of the overall calculated deviation for biological and heavy aromatic compounds from various methods

\begin{tabular}{|c|c|c|c|c|c|c|c|c|}
\hline \multirow[b]{2}{*}{ Group of solid solute } & \multirow[b]{2}{*}{ Data points } & \multicolumn{7}{|c|}{$\operatorname{AAD}(\%)$} \\
\hline & & I & II & III & IV & $\mathrm{V}$ & VI & VII \\
\hline Antioxidants & 28 & 6.99 & 2.87 & 6.81 & 3.51 & 3.66 & 1.34 & 1.44 \\
\hline Pollutants & 38 & 13.06 & 2.31 & 7.67 & 2.70 & 5.27 & 4.49 & 5.01 \\
\hline Steroids & 130 & 14.62 & 11.71 & 19.07 & 14.65 & 13.65 & 13.11 & 13.00 \\
\hline Vitamins & 59 & 17.36 & 11.57 & 14.21 & 11.92 & 11.12 & 10.91 & 12.49 \\
\hline Others & 91 & 12.70 & 8.33 & 14.14 & 10.69 & 11.83 & 9.74 & 9.41 \\
\hline Grand & 346 & 13.79 & 9.05 & 14.70 & 10.93 & 11.01 & 9.95 & 10.16 \\
\hline
\end{tabular}

I: $\mathrm{m}$-AD model with three parameters; II: $\mathrm{m}-\mathrm{AD}$ model with four parameters; III: Modified regular solution model with two adjustable parameters $\alpha$ and $\beta$ [4]; IV: Semi-empirical model of Chrastil [9], $\ln y_{2}=a_{0} \ln \rho_{1}+\left(a_{1} / T\right)+a_{2} ; \mathrm{V}$ : Semi-empirical model of Méndez-Santiago and Teja [10], $T \ln \left(y_{2} P\right)=a_{0}+a_{1} \rho_{1}+a_{2} T ;$ VI: Semi-empirical model of Jiang et al. [11], $\ln y_{2}=a_{0} \rho_{1}+\left(a_{1} / T\right)+a_{2} \ln P+a_{3}$; VII: PR EOS with vdW2 mixing rule and the optimally fitted sublimation pressure, $\ln P^{\text {sub }}=A-B / T$, where $A$ and $B$ are taken as optimally fitted parameters.

tool for many kinds of phase equilibrium calculations.

\section{References}

[1] Cheng, J.S., Tang, M. and Chen, Y.P. (2003) "Correlation and comparison of the infinite dilution activity coefficient on aqueous mixtures from a modified excess free energy model", Fluid Phase Equilib., In final revision.

[2] Ashour, I., Almehaideb, R., Fateen, S.E. and Aly, G. (2000) "Representation of solid-supercritical fluid phase equilibria using cubic equations of state", Fluid Phase Equilib. 167, 41.

[3] Iwai, Y., Koga, Y., Fukuda, T. and Arai, Y. (1992) "Correlation of solubilities of high-boiling components in supercritical carbon dioxide using a solution model", J. Chem. Eng. Jpn 25, 757.

[4] Cheng, J.S., Tang, M. and Chen, Y.P. (2002) "Correlation of solid solubility for biological compounds in supercritical carbon dioxide: comparative study using solution model and other approaches", Fluid Phase Equilib. 194-197, 483.

[5] Bush, D. and Eckert, C.A. (1998) "Prediction of solid-fluid equilibria in supercritical carbon dioxide using linear solvation energy relationships", Fluid Phase Equilib. 150-151, 479.

[6] Aranovich, G.L. and Donohue, M.D. (1996) “A new model for lattice systems", J. Chem. Phys. 105, 7059.

[7] Yalkowsky, S.H. (1979) "Estimation of entropies of fusion of organic compounds", Ind. Eng. Chem. Fundam. 18, 108.

[8] Ely, J.F., Haynes, W.M. and Bain, B.C. (1989) "Isochoric (p, $\mathrm{V}_{\mathrm{m}}$ T) measurements on $\mathrm{CO}_{2}$ and on $\left(0.982 \mathrm{CO}_{2}+0.018 \mathrm{~N}_{2}\right)$ from 250 to $330 \mathrm{~K}$ at pressures to $35 \mathrm{MPa}^{\prime \prime}$, J. Chem. Thermodyn. 21, 879.

[9] Chrastil, J. (1982) "Solubility of solids and liquids in supercritical gases", J. Chem. Phys. 86, 3016.

[10] Méndez-Santiago, J. and Teja, A.S. (1999) "The solubility of solids in supercritical fluids", Fluid Phase Equilib. 158-160, 501.

[11] Jiang, C., Pan, Q. and Pan, Z. (1998) "Solubility behavior of solids and liquids in compressed gases", J. Supercrit. Fluids 12,1 .
[12] Cortesi, A., Kikic, I., Alessi, P., Turtoi, G. and Garnier, S. (1999) "Effect of chemical structure on the solubility of antioxidants in supercritical carbon dioxide: experimental data and correlation", J. Supercrit. Fluids 14, 139.

[13] Macnaughton, S.J. and Foster, N.R. (1994) "Solubility of DDT and 2,4-D in supercritical carbon dioxide and supercritical carbon dioxide saturated with water", Ind. Eng. Chem. Res. 33, 2757.

[14] Madras, G., Erkey, C. and Akgerman, A. (1993) "A new technique for measuring solubilities of organics in supercritical fluids", J. Chem. Eng. Data 38, 422.

[15] Yun, S.L.J., Liong, K.K., Gurdial, G.S. and Foster, N.R. (1991) "Solubility of cholesterol in supercritical carbon dioxide", Ind. Eng. Chem. Res. 30, 2476.

[16] Alessi, P., Cortesi, A., Kikic, I., Foster, N.R., Macnaughton, S.J. and Colombo, I. (1996) "Particle production of steroid drugs using supercritical fluid processing", Ind. Eng. Chem. Res. 35, 4718.

[17] Kosal, E., Lee, C.H. and Holder, G.D. (1992) "Solubility of progesterone, testosterone, and cholesterol in supercritical fluids", J. Supercrit. Fluids 5, 169.

[18] Wong, J.M. and Johnston, K.P. (1986) "Solubilization of biomolecules in carbon dioxide based supercritical fluids", Biotechnol. Prog. 2, 29.

[19] Johannsen, M. and Brunner, G. (1997) "Solubilities of the fatsoluble vitamins $\mathrm{A}, \mathrm{D}, \mathrm{E}$ and $\mathrm{K}$ in supercritical carbon dioxide", J. Chem. Eng. Data 42, 106.

[20] Coutsikos, P., Magoulas, K. and Tassios, D. (1997) "Solubilities of p-quinone and 9,10-anthraquinone in supercritical carbon dioxide", J. Chem. Eng. Data 42, 463.

[21] Uchiyama, H., Mishima, K., Oka, S., Ezawa, M. and Ide, M. (1997) "Solubilities of flavone and 3-hydroflavone in supercritical carbon dioxide", J. Chem. Eng. Data 42,570 .

[22] Schmitt, W.J. and Reid, R.C. (1986) "Solubility of monofunctional organic solids in chemically diverse supercritical fluids", J. Chem. Eng. Data 31, 204.

[23] Ting, S.S.T., Macnaughton, S.J., Tomasko, D.L. and Foster, N.R. (1993) "Solubility of naproxen in supercritical carbon dioxide with and without cosolvents", Ind. Eng. Chem. Res. 32, 1471. 
Copyright $\odot 2003$ EBSCO Publishing 